\title{
A Localization Method for First and Second Heart Sounds Based on Energy Detection and Interval Regulation
}

\author{
Se Dong Min* and Hangsik Shin ${ }^{\dagger}$
}

\begin{abstract}
The present study suggests a localization method for the first $\left(S_{1}\right)$ and the second $\left(S_{2}\right)$ feature of heart sounds, based on an algorithm involving frequency filtering, energy detection, and interval regulation. Localization accuracy was evaluated by comparing the algorithm with the traditional Hilbert transform-based localization method. Results show that the sensitivity and the positive predictivity value of proposed method, respectively, were $97.27 \%$ and $99.94 \%$ in $S_{1}$ detection and $94.99 \%$ and $100 \%$ in $S_{2}$ detection.
\end{abstract}

Keywords: Energy detection, Heart sound, Hilbert transform, Interval regulation, Localization, Phonocardiogram (PCG)

\section{Introduction}

Auscultation, the action of listening to the internal sounds of the body using a stethoscope, is a simple and effective way to diagnosis of cardiac dysfunction and cardiovascular disease. Moreover, auscultation has been widely used for fundamental cardiac diagnoses because of its non-invasive and simple diagnostic characteristics. The major purpose of auscultation is listening to the heart sound caused by the closing of the heart valves. Heart sound contains many signals related to magnitude and frequency content, splitting, and the presence of additional systolic or diastolic murmur components. There are two dominant features of heart sounds: the first heart sound $\left(S_{1}\right)$ and the second heart sound $\left(S_{2}\right)$. $S_{1}$ reflects the closure of mitral and the tricuspid valves, and $S_{2}$ marks the closure of the aortic and pulmonary valves.

Heart sounds are usually investigated with $S_{1}$ and $S_{2}$ analysis. Because $S_{1}$ and $S_{2}$ occur at the onset of ventricular contraction and the end of ventricular systole, respectively, the left ventricular ejection time (LVET) and the preejection period (PEP) have been estimated by heart sound analysis [1]. Moreover, the diagnosis of valve dysfunction and hemodynamic research are widely known applications of heart sound analysis. For example, cardiac valve disorder has been classified by a neural network classifier [2] and blood pressure has been estimated with pattern analysis [3] using heart sounds. Furthermore, the relationship between $S_{2}$ and aortic blood pressure has been shown [4]. Although heart sounds are a useful tool clinically, they are hard to analyze from the non-stationary characteristic. That is, making a cardiac disorder diagnosis based on heart sounds

$\dagger \quad$ Corresponding Author: Dept. of Biomedical Engineering, Chonnam National University, Korea. (hangsik.shin@jnu.ac.kr)

* Dept. of Medical IT Engineering, Soonchunhyang University, Korea. (sedongmin@sch.ac.kr)

Received: February 14, 2014; Accepted: Mary 12, 2015 is still a considerable challenge [5-12]. Thus, the accurate localization of $S_{1}$ and $S_{2}$ is an important issue for heart sound analysis and clinical applications.

Previous localization methods for heart sounds can be summarized as filtering, transform-based de-noising, and feature classification. In particular, wavelet de-noising has been researched to enable accurate heart sound localization [13-15]. There have been several approaches to $S_{1}$ and $S_{2}$ localization, such as spectral tracking [16], Shannon energy $[17,18]$, boundary modification method [19], time-delayed neural network [20], ECG-gated method [7], entropy based detection [21], homomorphic filtering [8], complexity based segmentation [22], hidden Markov model [23], and wavelet transform [24, 25]. Recently, wavelet de-noising and ECGgated peak-peaking methods have been improved by an ensemble average method for more accurate localization [26]. Also, in 2012, $S_{1} / S_{2}$ heart sounds detection based on music beat tracking has been researched [27].

Most heart sound localization methods are based on the energy of the features. A problem with energy-based detection methods is the low amplitude of $S_{1}$ and $S_{2}$ components; they can easily be buried in background noise, murmurs, or lung sounds, and they are not reflected in the energy envelope of the heart sound [22]. Moreover, it has been shown that the amplitude of the heart sound waveform is changed by respiration [28]; the amplitude of the heart sound waveform is attenuated by inspiration. The ECG-gated localization method, a commonly used technique for the localization, is based on ECG features, such as the R-wave. ECG-gated localization provides relatively stable localization [26]. However, ECG should also be measured as a reference in the ECG-gated localization method. Moreover, for a variety of pathological conditions, there may be detection errors because of inconsistencies between the electrical and mechanical activities of the heart [29]. 
The aim of the present study is the localization of $S_{1}$ and $S_{2}$ in heart sounds. In this study, features were discriminated from a phonocardiogram only. To improve localization performance, cascade integrator comb (CIC) filtering and an energy- and temporal-based localization method were developed. An experiment was performed in spontaneous and a $0.1 \mathrm{~Hz}$ or $0.25 \mathrm{~Hz}$ respiration-controlled environment to produce practical respiration effects and distortion. Statistical methods were used for a quantitative evaluation of the proposed algorithm.

\section{Experimental Setup}

\subsection{Dataset}

Eighteen young, healthy subjects (11 males, 7 females, mean age is 24.1 , range $17-30$ years old) participated. Prior to the experiment, the subjects were requested to provide information on their physical condition. None reported any taking medicine or signs of cardiovascular or respiratory disease. Each experiment was performed in a typical sports medicine laboratory at ambient room temperature, from 11 am to $6 \mathrm{pm}$. Drinking and smoking were prohibited during 24 hours and 2 hours before experiments, respectively. Anthropometric data, such as height and weight, were measured and are summarized in Table 1.

\subsection{Devices and software}

Heart sounds were recorded with a MP150 (Biopac Systems, inc., CA, USA), DA 100C acquisition module, and SS17L contact microphone. Heart sounds were measured on the chest surface between the $3^{\text {rd }}$ and $4^{\text {th }}$ ribs, and the ECG was measured on the chest surface with a Lead II configuration (Fig. 1). Acknowledge 3.9.1 (BIOPAC, USA) was used for real-time monitoring and data storage.

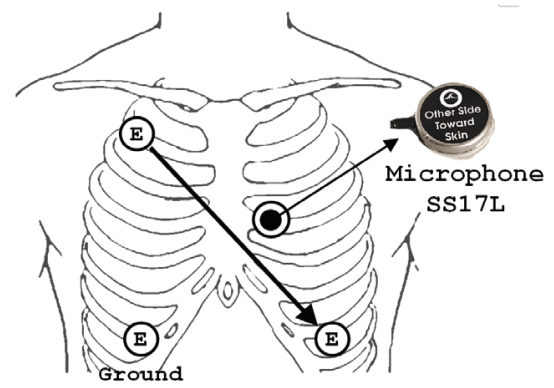

Fig. 1. Location of heart auscultation and ECG measurements

For signal conditioning, localization, and statistical analyses, MATLAB 2008b (Mathworks, Inc., Natick, MA, USA) was used and an HEM-907(Omron, Kyoto, Japan) was used for blood pressure measurements.

\subsection{Experimental protocol}

Heart sounds were measured, including spontaneous and controlled respiration effects. Respiration was controlled at $0.1 \mathrm{~Hz}$ (low rate) and $0.25 \mathrm{~Hz}$ (high rate) in a supine position using a metronome. In the sitting position, there was no respiration controlling. Experiments were carried out sequentially in the following order: spontaneous, 0.1 $\mathrm{Hz}$ controlled, and $0.25 \mathrm{~Hz}$ controlled respiration in the supine position, and spontaneous respiration in the sitting position. Subjects had a 2-min rest period between experiments. Data were sampled at a $1 \mathrm{kHz}$ sampling rate for a 5 -min period.

\section{Signal Conditioning and Localization}

\subsection{Cascade integrator comb filter}

CIC filtering has been used for biomedical signal conditioning and feature enhancement [29]. A CIC filter,

Table 1. Subject demographic data $\left({ }^{\mathrm{a}}\right.$ Systolic Blood Pressure, ${ }^{\mathrm{b}}$ Diastolic blood pressure, ${ }^{\mathrm{c}}$ Heart rate (beats per minute)

\begin{tabular}{|c|c|c|c|c|c|c|c|c|}
\hline Subject & Gender & Age & Height $(\mathrm{cm})$ & Weight $(\mathrm{kg})$ & BMI $\left(\mathrm{kg} / \mathrm{m}^{2}\right)$ & $\mathrm{SBP}^{\mathrm{a}}(\mathrm{mmHg})$ & $\mathrm{DBP}^{\mathrm{b}}(\mathrm{mmHg})$ & $\mathrm{HR}(\mathrm{bpm})^{\mathrm{c}}$ \\
\hline 1 & male & 29 & 185 & 103 & 30.1 & $124 \pm 5.7$ & $70 \pm 6.8$ & $69.70 \pm 7.23$ \\
\hline 2 & male & 30 & 172 & 81 & 27.6 & $139 \pm 4.8$ & $81 \pm 4.9$ & $82.80 \pm 6.94$ \\
\hline 3 & male & 29 & 178 & 68 & 21.4 & $127 \pm 13.7$ & $73 \pm 6.5$ & $78.10 \pm 2.22$ \\
\hline 4 & male & 28 & 175 & 78 & 25.3 & $122 \pm 9.4$ & $71 \pm 2.2$ & $61.95 \pm 9.05$ \\
\hline 5 & male & 26 & 175 & 63 & 20.6 & $110 \pm 3.5$ & $66 \pm 2.4$ & $69.02 \pm 3.26$ \\
\hline 6 & female & 26 & 161 & 57 & 22.2 & $100 \pm 1.9$ & $63 \pm 2.0$ & $57.04 \pm 0.78$ \\
\hline 7 & female & 23 & 162 & 56 & 21.2 & $101 \pm 3.7$ & $67 \pm 5.3$ & $64.87 \pm 4.07$ \\
\hline 8 & female & 21 & 161 & 60 & 23.1 & $107 \pm 4.6$ & $65 \pm 3.4$ & $79.73 \pm 1.80$ \\
\hline 9 & male & 29 & 177 & 71 & 22.6 & $126 \pm 10.7$ & $72 \pm 8.1$ & $65.65 \pm 3.29$ \\
\hline 10 & male & 24 & 171 & 68 & 23.1 & $135 \pm 7.7$ & $72 \pm 7.4$ & $68.82 \pm 3.78$ \\
\hline 11 & male & 17 & 171 & 65 & 22.2 & $131 \pm 8.4$ & $67 \pm 8.9$ & $70.61 \pm 2.97$ \\
\hline 12 & female & 22 & 159 & 47 & 18.5 & $103 \pm 6.6$ & $68 \pm 4.7$ & $78.98 \pm 1.07$ \\
\hline 13 & male & 25 & 174 & 77 & 25.7 & $119 \pm 9.3$ & $72 \pm 5.7$ & $69.21 \pm 1.74$ \\
\hline 14 & female & 22 & 163 & 55 & 20.5 & $107 \pm 3.1$ & $74 \pm 5.6$ & $80.14 \pm 6.39$ \\
\hline 15 & male & 18 & 175 & 83 & 26.9 & $134 \pm 9.1$ & $75 \pm 6.8$ & $63.70 \pm 5.16$ \\
\hline 16 & male & 23 & 172 & 71 & 24.2 & $140 \pm 9.1$ & $77 \pm 12.5$ & $56.46 \pm 4.22$ \\
\hline 17 & female & 19 & 164 & 63 & 23.6 & $110 \pm 2.9$ & $69 \pm 5.1$ & $57.60 \pm 5.30$ \\
\hline 18 & female & 21 & 160 & 60 & 23.6 & $120 \pm 4.5$ & $75 \pm 5.7$ & $61.49 \pm 3.67$ \\
\hline
\end{tabular}


which is a linear-phase filter, was implemented for signal conditioning of the heart sounds. Linear phase-filtering, which prevents phase and timing distortion, is important because the heart sound has non-stationary characteristic. A CIC filter is composed of a high-pass and a low-pass filter and its transfer function is described in (1) and (2). $\mathrm{N}_{\mathrm{hpf}}$, $\mathrm{N}_{\text {lpf }}$, and $\mathrm{M}$ indicate the high-pass filter order, low-pass filter order, and number of cascaded stages, respectively; their values were 16,8 , and 2 , respectively, in the filter design. $\mathrm{N}_{\text {hpf }}, \mathrm{N}_{\mathrm{lpf}}$, and $\mathrm{M}$ had a $43.2 \mathrm{~Hz}$ center frequency and pass-band between $26.6 \mathrm{~Hz}$ and $63 \mathrm{~Hz}$ ( $3 \mathrm{~dB}$ point). The frequency characteristics of the CIC filter are presented in Fig. 2(a).

$$
\begin{aligned}
& H_{h p f}(z)=z^{-D}-\left(\frac{1}{N_{h p f}} \cdot \frac{1-z^{-N_{h p f}}}{1-z^{-1}}\right)^{M}, \\
& D=\frac{N_{h p f}-1}{2} \cdot M \\
& H_{l p f}(z)=\left(\frac{1}{N_{l p f}} \cdot \frac{1-z^{-N_{l p f}}}{1-z^{-1}}\right)^{M}
\end{aligned}
$$

\subsection{Moving average filter}

A general heart sound is described with a high-frequency signal. However, specific features, such as $S_{1}$ and $S_{2}$, are decided by the value of the envelope. From the natural

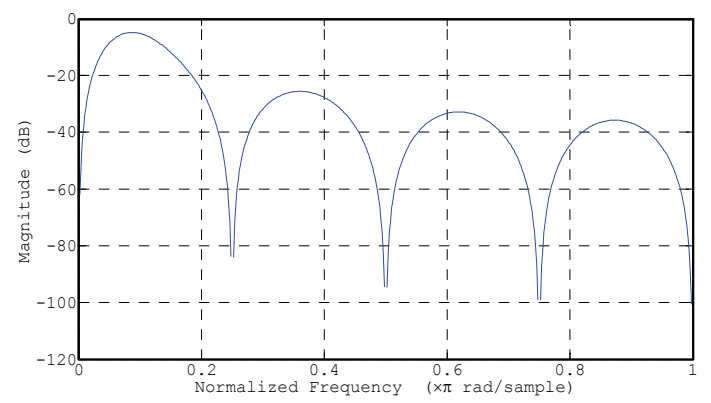

(a)

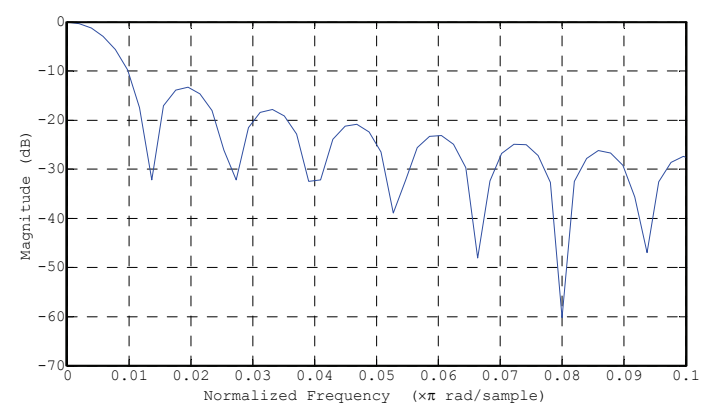

(b)

Fig. 2. Frequency characteristic of filters: (a) Cascade integrator comb filter. The center frequency is 43.2 $\mathrm{Hz}$ and the pass-band is 26.6 to $63 \mathrm{~Hz}$; (b) Moving average filter (cut-off frequency is $\sim 3 \mathrm{~Hz}$ ) characteristics of heart sounds, the envelope detected by the Hilbert transform contains many tiny fluctuations that could have an effect on specific feature extraction. These fluctuations are also regarded as high frequency noise in feature extraction; thus, a moving-average filter (MAF) was used for envelope smoothing as a kind of lowpass filter. The frequency response of MAF is shown in (3). $\mathrm{L}$ is the MAF length and it was of $0.15 \mathrm{~s}$ duration (cutoff frequency is $\sim 3 \mathrm{~Hz}$ ). Fig. 2(b) shows the frequency characteristics of MAF.

$$
H_{M A F}(z)=\frac{1}{L} \cdot \frac{1-z^{-L}}{1-z^{-1}}
$$

\subsection{Hilbert transform}

The Hilbert transform is proper for time-varying signal analysis, used frequently for envelope detection with heart sounds [31]. The analytic function of $z[n]$ contains a realvalue function $x[n]$ and a complex value function $\tilde{x}[n]$, which is the Hilbert transform pair of $x[n]$. The analytical signal and envelope detection equations are described in (4) and (5), respectively.

$$
\begin{gathered}
z[n]=x[n]+j \tilde{x}[n] \\
\text { Envelope }[n]=\sqrt{x^{2}[n]+\tilde{x}^{2}[n]}
\end{gathered}
$$

\section{4 $S_{1}$ and $S_{2}$ Localization}

The heart sound signal is processed sequentially, with CIC filtering, the Hilbert transformation, and movingaverage filtering. Then, the $S_{1}$ and $S_{2}$ peaks are detected from the filtered envelope of the heart sound. $S_{1}$ peaks were detected by a zero-crossing method, based on signal derivation. For $S_{2}$ localization, an energy based detection method was used within the $S_{1}$ intervals. The $S_{1}$-gated window was determined from the $(\mathrm{n}-1)^{\text {th }}$ position of $S_{1}$ $\left(\mathrm{PS} 1_{\mathrm{n}-1}\right)$ to the $\mathrm{n}^{\text {th }}$ position of $S_{1}\left(\mathrm{PS} 1_{\mathrm{n}}\right)$. A 0.2 -s refractory period was also defined to reduce misdetection for the former and latter $S_{1}$ location. Thus, the scanning window

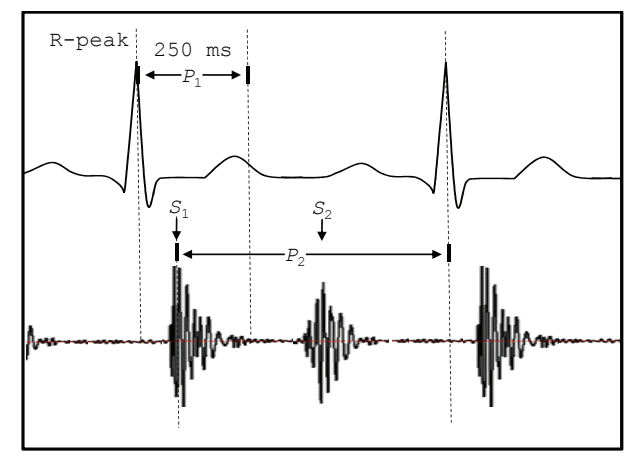

Fig. 3. Localization method of the reference $S_{1}$ and $S_{2}$ positions 
had a range between $P S 1_{n-1}+0.2$ and $P S 1_{n}-0.2$. Within the scanning window, $S_{2}$ was localized by taking the maximum value of the energy waveform of the heart sound.

The reference peak position was detected by an ECGgated detection method and the Hilbert transformation. The $S_{1}$ location was decided within a $250 \mathrm{~ms}$ detection window (P1) following the R peak of the ECG waveform. The $S_{2}$ location was detected by the maximum energy within the specified range $\left(\mathrm{P}_{2}\right) . \mathrm{P}_{2}$ starts from the $S_{1}$ location to the next R peak of the ECG. Each detected $S_{1}$ and $S_{2}$ was corrected manually (Fig. 3).

\subsection{Peak correction}

The envelope of the heart sound has two distinguishable peaks, at $S_{1}$ and $S_{2}$. In using time difference-based classification method, the detection of both $S_{1}$ and $S_{2}$ should precede $S_{1}$ and $S_{2}$ classification. The detected peaks are typically located at the $S_{1}$ and $S_{2}$ positions. However, redundant peaks or missed peaks may still remain. In the pre-processing stage of $S_{1}$ localization, mis-detected peak correction is directly related to detection accuracy. The proposed localization method is based on time difference characteristics between $S_{1}$ and $S_{2}$. Because the $S_{2}-S_{1}$ interval is generally longer than the $S_{1}-S_{2}$ interval, the difference in peak time interval (PTI) can be described with an up-and-down curve. If we assume that every peak was detected correctly, then the PTI curve should be repeated with a regular up-and-down pattern. However, a PTI curve could show an irregular trend from missed or redundant peaks. In the missed peak case, PTI becomes higher than the normal upper limit (6) and redundant peaks causes a lower PTI value than the other lower peaks (7). To remove these irregularities, a peak correction method was used. Peak correction was carried out with two processes: False Positive Peak correction (FPPC) and False Negative Peak correction (FNPC). FPP correction modifies PTI values using (8); a redundant peak time interval at the nth PTI, PTIn, is removed by comparison between the former and the latter PTI value. In the FNP correction, in contrast, a new PTI is added using (9). The estimated $S_{1}$ and $S_{2}$ intervals are decided by average values of $50 \%$ higher or lower.

$$
\begin{aligned}
& P T I_{n}>\text { mean }(P T I)+2.5 \operatorname{std}(P T I) \\
& P T I_{n}<\text { mean }(P T I)-\operatorname{std}(P T I) \\
& \begin{cases}P T I_{n-1}=P T I_{n-1}+P T I_{n} & , P T I_{n-1}<P T I_{n+1} \\
P T I_{n+1}=P T I_{n+1}+P T I_{n} & , P T I_{n-1}>P T I_{n+1}\end{cases} \\
& \begin{cases}P T I_{n+1}=P T I_{n+1}-E I_{s 1} & , P T I_{n-1}<P T I_{n+1} \\
P T I_{n+1}=P T I_{n+1}-E 1_{s 2} & , P T I_{n-1}>P T I_{n+1}\end{cases}
\end{aligned}
$$

Moreover, both $S_{1}$ and $S_{2}$ should be detected after the signal conditioning procedures and classified with PTI analysis for $S_{1}$ localization. Fig. 4 shows the PTI analysis

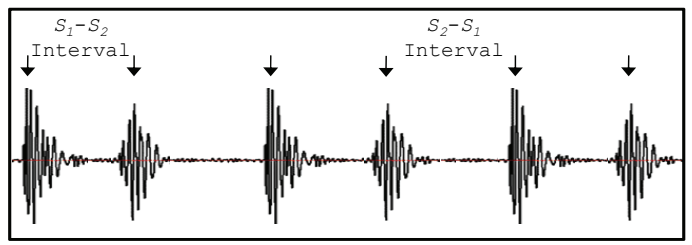

(a)

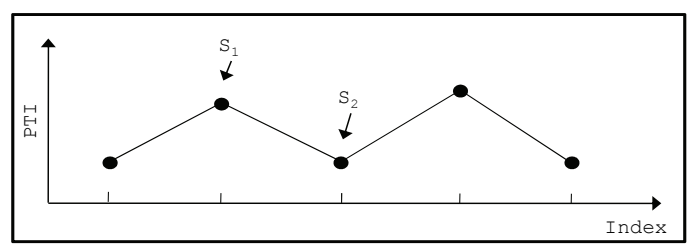

(b)

Fig. 4. Peak time interval (PTI) analysis and S1 localization: (a) Detected peaks and intervals between $\mathrm{S}_{1}$ and $\mathrm{S}_{2}$;

(b) PTI and $\mathrm{S}_{1}$ localization. $\mathrm{S}_{1}$ is located at the upto-down breakpoint.

procedure. PTI can be represented with an up-and-down plot in the general case, and the up-to-down breakpoint indicates the $S_{1}$ location.

\subsection{Replace, add, and delete algorithm}

As noted previously, a representative detection problem can be summarized with FPP and FNP. In $S_{1}$ classification, a similar problem occurs. Typically, FNP means missed peaks or confusing $S_{1}$ and $S_{2}$. Moreover, FPP means that there are redundant peaks around the actual $S_{1}$ location. To improve this detection problem, a replace, add, and delete (RAD) algorithm is proposed.

Replace, add, and delete procedures are defined by PTI range. In the replace procedure, when $S_{2}$ is detected as a substitute of $S_{1}$, PTI is between Limit $_{\mathrm{h} 1}$ and Limit $_{\mathrm{h} 2}$ (Limit $_{\mathrm{h} 1}$ $<$ PTI $<$ Limit $_{\mathrm{h} 2}$ ). If PTI is longer than the replace interval $\left(\right.$ Limit $\left._{\mathrm{h} 2}<\mathrm{PTI}\right)$, it is regarded as an add procedure. The delete procedure is invoked when PTI is shorter than Limit $_{1}$ $\left(\right.$ Limit $_{1}>$ PTI). Classification criteria, Limit $t_{\mathrm{h} 1}$, Limit $_{\mathrm{h} 2}$, and Limit $_{\mathrm{l}}$, are determined using (10), (11) and (12), respectively, with empirical constant, and they are adaptively changed by the previous $S_{1}$ interval.

$$
\begin{aligned}
& \left\{\begin{array}{l}
\text { Limit }_{1}=1.2 M B R_{\text {TOTAL }} \\
\text { Limit }_{h 1}=1.4 M B R_{\text {prev }}
\end{array},\right. \text { if previous peaks are not exist. } \\
& \left\{\begin{array}{l}
\text { Limit }_{h 2}=1.8 M B R_{\text {TOTAL }} \\
\text { Limit }_{h 2}=1.8 M B R_{\text {prev }}
\end{array},\right. \text { if previous peaksare not exist. } \\
& \left\{\begin{array}{l}
\text { Limit }_{l}=0.4 M B R_{\text {TOTAL }} \\
\text { Limit }_{l}=0.5 M B R_{\text {prev }}
\end{array},\right. \text { if previous peaksare not exist. }
\end{aligned}
$$

Fig. 5 shows three detection problems and correction methods such as 'Replace', 'Add' and 'Delete' algorithm. 


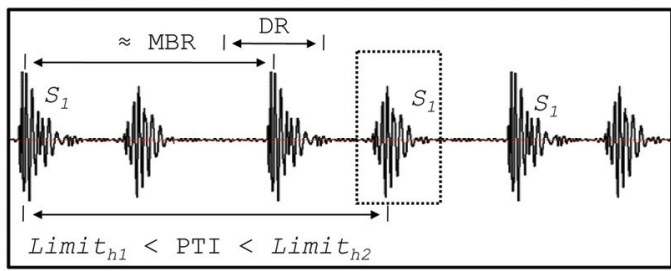

(a)

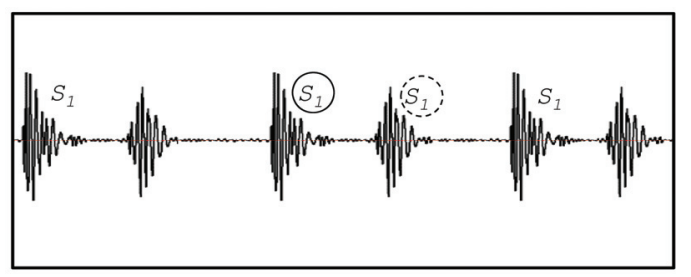

(b)

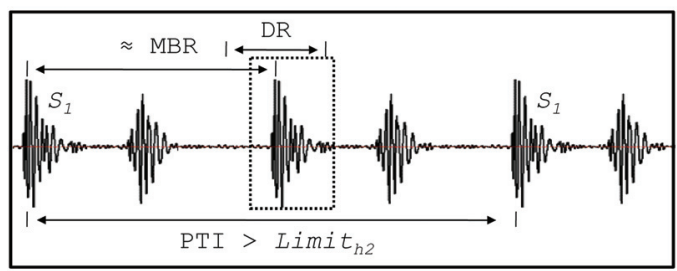

(c)

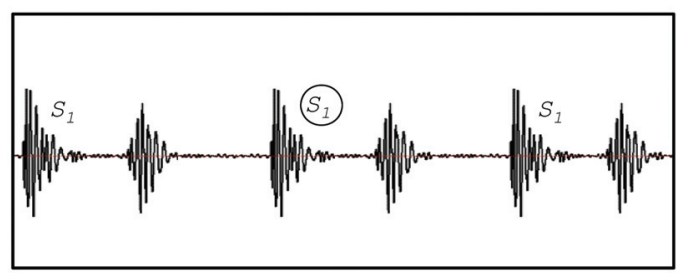

(d)

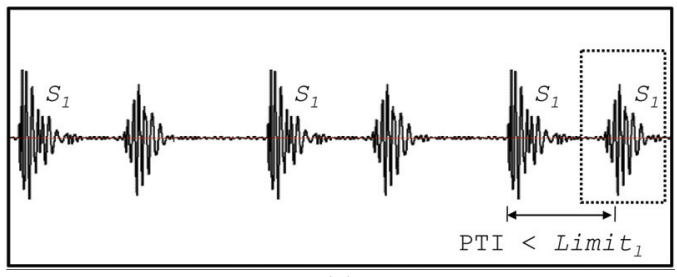

(e)

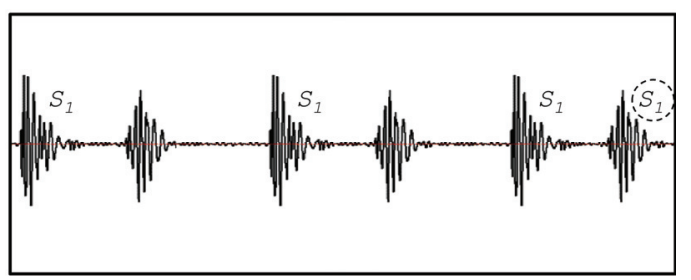

(f)

Fig. 5. $S_{1}$ detection problems and results of the Replace, Add, and Delete (RAD) algorithm. A solid circle indicates a newly added $\mathrm{S}_{1}$ and a dotted circle indicates a deleted $\mathrm{S}_{1}$ : (a) False detection of $\mathrm{S}_{1}$; (b) The correction result by the 'Replace' procedure of (a); (c) Detection failure of $\mathrm{S}_{1}$; (d) The corrected result by the 'Add' procedure of (c); (e) Duplicated $\mathrm{S}_{1}$ detection; (f) The result of the 'Delete' procedure of (e).
In Fig. 5, dotted box shows the detection problem with $S_{1}$ detection. Fig. 5(a) indicates a false detection of $S_{1}$ when PTI is between Limit $_{\mathrm{h} 1}$ and Limit $\mathrm{h}_{\mathrm{h} 2}$. Fig. 5(b) shows the corrected result of the 'replace' procedure for (a). A new $S_{1}$ is detected with the mean beat rate (MBR) and detection range (DR). MBR is decided with the average value of the previous $S_{1}$ time difference and DR is $0.2 \mathrm{~s}$, empirically. The second detection problem is shown in Fig. 5(c). A $S_{1}$ is missed when PTI is longer than Limit $t_{\mathrm{h} 2}$. Fig. 5(d) is the result of the 'add' procedure. In add procedure, $S_{1}$ is added by the same rule of replace method. However, in add procedure, the next $S_{1}$ would not be deleted because a $S_{1}$ is missed. The last detection problem is represented in Fig. 5(e). $S_{1}$ is detected again when PTI is shorter than Limit . Fig. 5(f) shows the result of the 'delete' procedure. In this procedure, the latter $S_{1}$ is deleted between two adjacent $S_{1}$, and the former $S_{1}$ is retained.

Fig. 6 shows a flowchart of the RAD algorithm and $S_{2}$

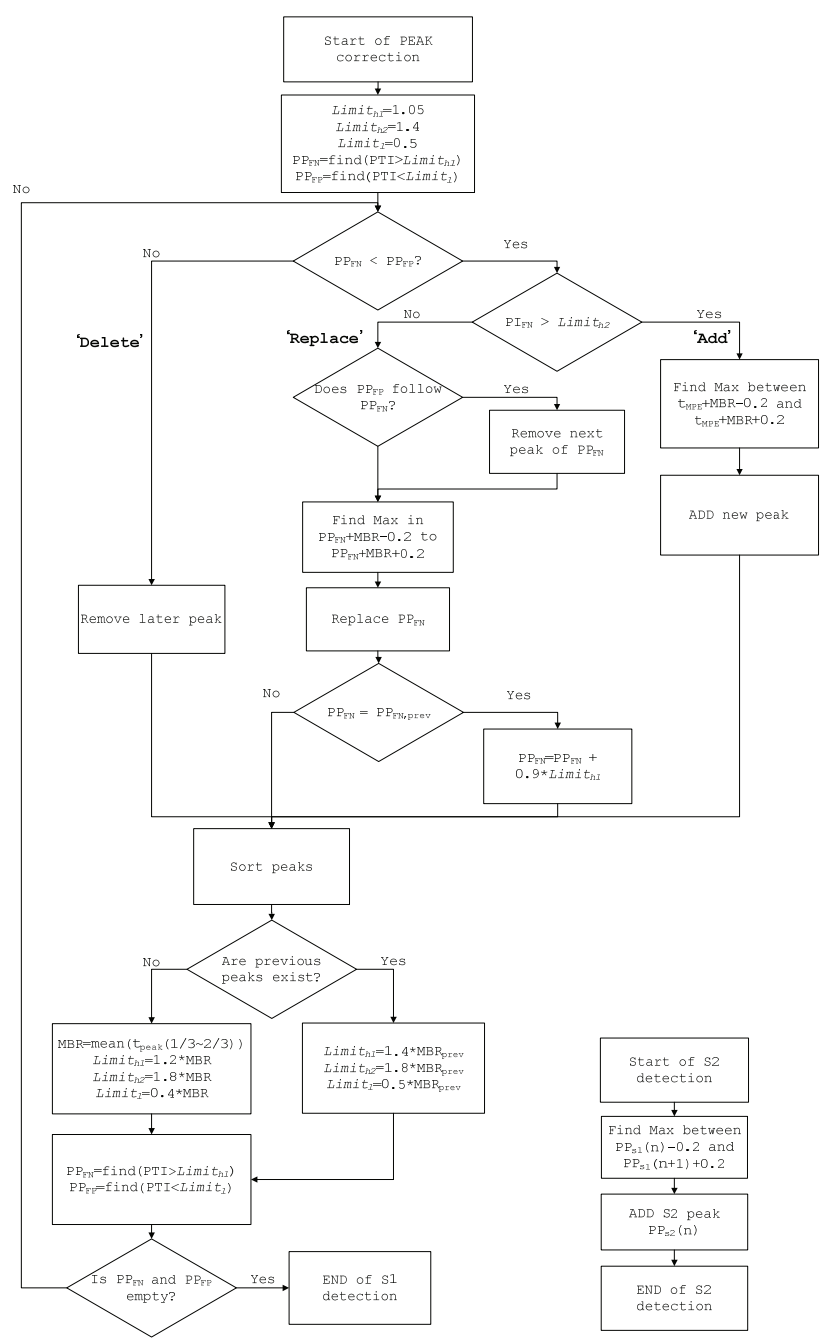

Fig. 6. Flowchart of the RAD algorithm and $S_{2}$ detection: (a) RAD algorithm for $S_{1}$ correction; (b) $S_{2}$ localization procedure. $\mathrm{MBR}=$ mean beat rate, $\mathrm{PI}=$ peak interval, $\mathrm{PP}_{\mathrm{n}}=n$-th peak position. 
detection. The RAD algorithm is repeated until every $S_{1}$ is corrected. The $S_{2}$ localization depends on $S_{1}$ detection and it is represented in Fig. 5(b). $S_{2}$ is determined by the maximum value position between two adjacent $S_{1}$.

\section{Evaluation}

For localization performance evaluation, the true positive (TP), false positive (FP), false negative (FN) and failed detection (FD) were calculated. Then, we derived the sensitivity (SE), positive predictivity ( $+\mathrm{P})$, and failed detection rate (FDR) to evaluate the accuracy of the detection algorithm. Detected peaks were verified with a 200-ms approval range from the reference peak positions that were obtained by ECG-gated detection. Sensitivity was calculated using (13), +P using (14), and FDR using (15). Evaluations were carried out for both $S_{1}$ and $S_{2}$ localizations.

$$
\text { Sensitivity }(\mathrm{SE})=\frac{\text { True Posituve }}{\text { True Positive }+ \text { False Negative }} \times 100(\%)
$$

Positive predictivity $(+\mathrm{P})$

$$
\begin{aligned}
& =\frac{\text { True Posituve }}{\text { True Positive }+ \text { False Positive }} \times 100(\%) \\
& \text { Failed detection rate }(\text { FDR })=\frac{\text { Failed Detection }}{\text { Total Peaks }} \times 100(\%)
\end{aligned}
$$

\section{Results}

\subsection{Signal conditioning}

The signal conditioning results are shown in Fig. 7. Fig. 7 (a) represents the original heart sound waveform, and (b), (c), and (d) are the CIC-filtered signal, Hilbert-transformed signal, and moving-average-filtered signal, respectively. In Fig. 7(d), the detected $S_{1}$ and $S_{2}$ peaks are described.

\subsection{Peak correction and classification}

The initially detected peaks after signal conditioning are processed with the FPPC and FNPC methods. The FPPC and FNPC results are represented in Fig. 8. Initially detected peaks are shown in Fig. 8(a), and Fig. 8(b) shows the PTI of (a). FPP and FNP could be detected with detection criteria (1) and (2), respectively, described as relatively low and high PTI values in Fig. 8(b). The corrected peak and its PTI are presented in Figs. 8(c) and 8(d), respectively. After peak correction, the $S_{1}$ and $S_{2}$ locations are decided at the up-or-down peaks of the PTI curve.

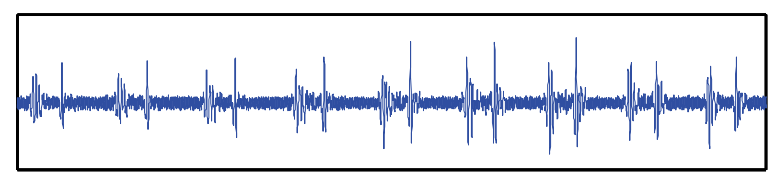

(a)

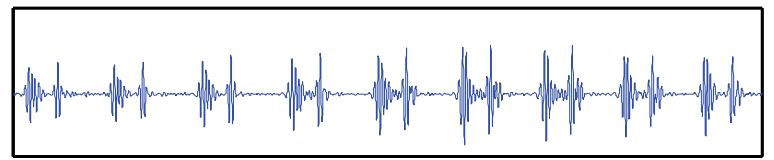

(b)

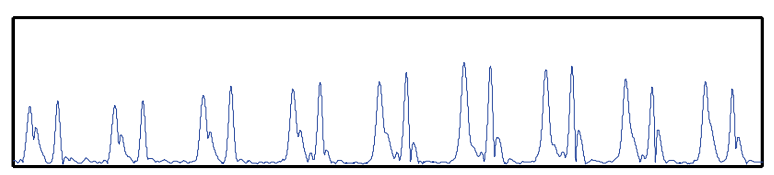

(c)

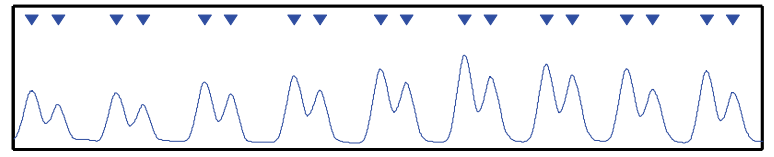

(d)

Fig. 7. Signal conditioning procedure and digital filtering: (a) Original heart sound; (b) CIC-filtered result (M $=2, \mathrm{~N}_{\mathrm{lpf}}=16, \mathrm{~N}_{\mathrm{hpf}}=32$ ); (c) Hilbert-transformation result; (d) Moving average integrator result (window size $=0.15 \mathrm{~s}) .{ }^{\prime} \boldsymbol{\nabla}$ ' indicates a detected peak.

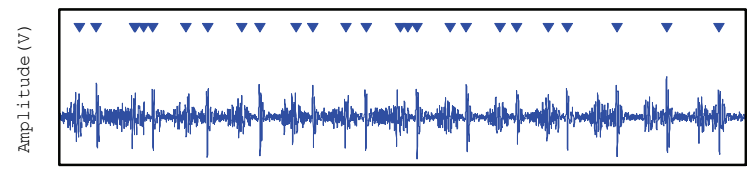

(a)

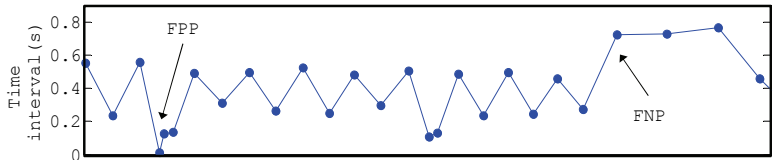

(b)

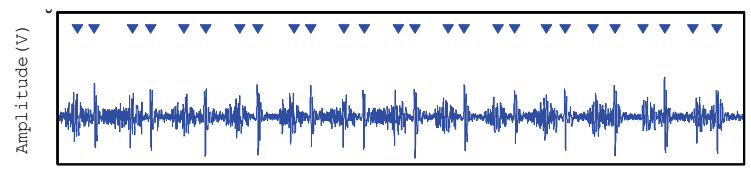

(c)

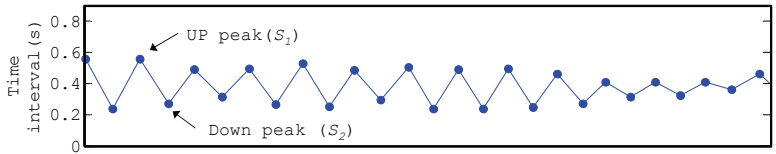

(d)

Fig. 8. Correction and classification of the detected peaks after signal conditioning: (a) Detected peaks by differential method; (b) Peak time intervals of (a). False positive peaks (FPP) and false negative peaks (FNP) were detected in this stage; (c) Corrected peaks. (d) Peak time intervals of (c). A longer time interval (UP peak) means a $S_{1}$ and shorter time interval (Down peak) means a $S_{2}$ peak. 


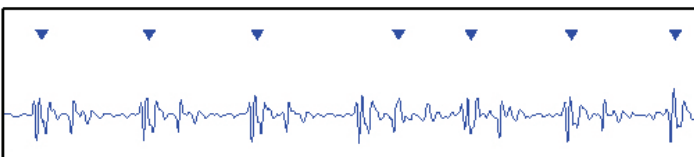

(a)

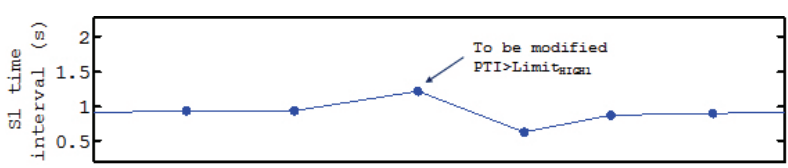

(b)

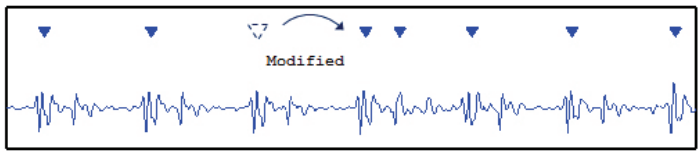

(c)

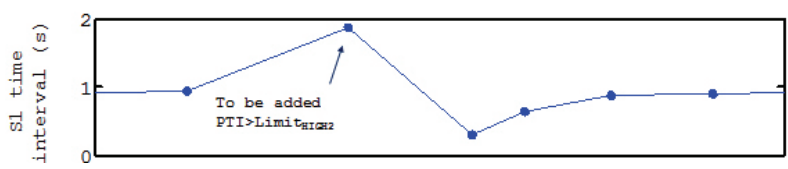

(d)

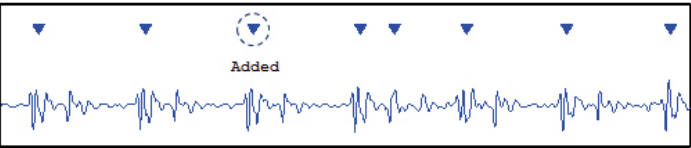

(e)

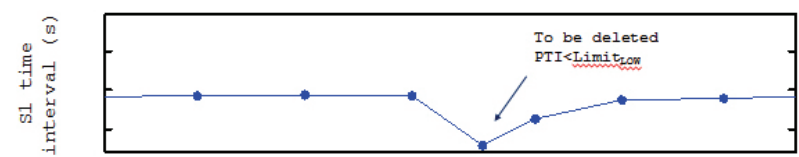

(f)

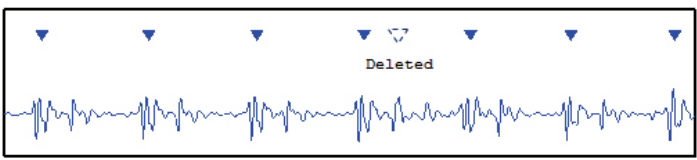

(g)

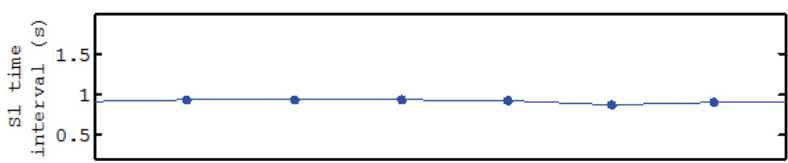

(h)

Fig. 9. An example of the $S_{1}$ correction method: (a) $S_{1}$ detection result before RAD algorithm. There is a misdetected peak between the $3^{\text {rd }}$ and $5^{\text {th }}$ peaks; (b) Peak time interval (PTI) of (a). It was found to be a PTI>Limit HIGH $_{\text {peak; }}$ (c) 'Replace' procedure; (d) PTI of (c); (e) 'Add' procedure; (f) PTI of (e); (g) 'Delete' procedure; (h) PTI of (g).

Table 2. Results evaluating the $S_{l}$ detection algorithm at each stage. The method consists of CIC filtering, peak correction (PC), and the replace, add, and delete (RAD) algorithm

\begin{tabular}{c|c|c|c|c|c|c|c|c|c|c}
\hline No. & Method & $\mathrm{RS}_{1}{ }^{a}$ (beats) & $\mathrm{DS}_{1}{ }^{\mathrm{b}}$ (beats) & $\mathrm{TP}$ (beats) & $\mathrm{FP}$ (beats) & FN (beats) & FD (beats) & SE (\%) & + P (\%) & FDR (\%) \\
\hline 1 & Hilbert+MAF & 27082 & 23783 & 23716 & 67 & 3366 & 3433 & 87.57 & 99.72 & 14.48 \\
\hline 2 & 1+CIC filter & 27082 & 25409 & 25397 & 12 & 1685 & 1697 & 93.78 & 99.95 & 6.68 \\
\hline 3 & $2+\mathrm{PC}$ & 27082 & 25647 & 25643 & 4 & 1439 & 1443 & 97.69 & 99.98 & 5.63 \\
\hline 4 & $3+\mathrm{RAD}$ & 27082 & 26358 & 26343 & 15 & 739 & 754 & 97.27 & 99.94 & 2.86 \\
\hline
\end{tabular}

${ }^{\mathrm{a}}$ Total number of reference $S_{1}$

${ }^{\mathrm{b}}$ Total number of detected peaks

Table 3. Results evaluating the $S_{2}$ detection algorithm at each stage. The method consists of CIC filtering, peak correction (PC), and the replace, add, and delete (RAD) algorithm

\begin{tabular}{c|c|c|c|c|c|c|c|c|c|c}
\hline No. & Method & $\mathrm{RS}_{2}{ }^{\mathrm{a}}$ (beats) & $\mathrm{RS}_{2}{ }^{\mathrm{b}}$ (beats) & $\mathrm{TP}$ (beats) & $\mathrm{FP}$ (beats) & $\mathrm{FN}$ (beats) & FD (beats) & SE (\%) & $+\mathrm{P}(\%)$ & FDR (\%) \\
\hline 1 & Hilbert+MAF & 27082 & 22780 & 22780 & 0 & 4358 & 4358 & 83.94 & 100 & 19.13 \\
\hline 2 & 1+CIC filter & 27082 & 24394 & 24394 & 0 & 2697 & 2697 & 90.04 & 100 & 11.06 \\
\hline 3 & $2+\mathrm{PC}$ & 27082 & 24824 & 24824 & 0 & 2264 & 2264 & 91.64 & 100 & 9.12 \\
\hline 4 & $3+\mathrm{RAD}$ & 27082 & 25740 & 25740 & 0 & 1357 & 1357 & 94.99 & 100 & 5.27 \\
\hline
\end{tabular}

${ }^{a}$ Total number of reference $S_{2}$.

${ }^{\mathrm{b}}$ Total number of detected peaks.

\subsection{Replace, Add, and delete correction}

Classified $S_{1}$ peaks were also corrected with the RAD algorithm. Fig. 9 shows an example of $S_{1}$ correction with the RAD algorithm. Fig. 9(a) and (b) show the initially detected $S_{1}$ and its PTI. RAD correction was carried out using (8), (9), and (10). Figs. 9(c) and (d), Figs. 9(e) and (f), and Figs. 9(g) and (h) show the replace, add, and delete procedures and the PTI, respectively.

\subsection{Statistical evaluation}

In the statistical analysis, $\mathrm{SE},+\mathrm{P}$, and FDR were calculated to evaluate the classification performance at each stage. The results of $S_{1}$ and $S_{2}$ localization are presented in Tables 2 and 3. In $S_{1}$ localization, SE was increased, $87.57 \%$ to $97.27 \%$, and $+\mathrm{P}$ was increased, $99.72 \%$ to $99.94 \%$. FDR decreased, $14.48 \%$ to $2.86 \%$. Except for slight fluctuation in $+\mathrm{P}$, each performance evaluation index was increased in every correction stage.

Performance for $S_{2}$ localization was evaluated in the same way as $S_{1}$. In $S_{2}$ localization, SE increased, $83.94 \%$ to $94.9 \%$, and $+\mathrm{P}$ was $100 \%$ in each case. FDR decreased, $19.13 \%$ to $5.27 \%$. Each performance evaluation index of $S_{2}$ also increased in every correction stage. Overall localization performance for $S_{1}$ was better than for $S_{2}$ 


\section{Conclusion}

\subsection{Performance evaluation}

The proposed method was validated by a quantitative study with 27,082 pulses, posture changes, and respiratory variation. Localization performance was most improved by CIC filtering; SE and FDR were improved by $6.21 \%$ and $7.8 \%$, respectively, in the $S_{1}$ case. In the $S_{2}$ case, they were also improved, by $6.1 \%$ and $8.07 \%$. +P was not changed significantly. Remaining misdetected peaks after frequency domain-filtering were corrected by peak correction and the RAD algorithm. These algorithms improved FDR by $1.05 \%$ and $1.95 \%$ in the $S_{l}$ case. These results could be considered as slight improvement; however, it is meaningful to recover from missing or extremely distorted peaks. Because $S_{2}$ detection depends largely on $S_{1}$ results, $S_{2}$ detection performance had a lower value than $S_{l}$ results; however, +P was always $100 \%$.

\subsection{Limitation}

A time-dependent classification algorithm is hard to adapt to an irregular heartbeat. For example, the heart sound of an arrhythmia patient could generate more errors because a correction criterion is affected by the previous PTI and specific constant. An error could even be propagated in certain cases.

In $S_{2}$ localization, the $S_{2}$ detection rate depends on the $S_{1}$ detection result. Thus, failed detection of $S_{1}$ could be a reason of the failure of $S_{2}$ localization. The proposed algorithm focuses on a post-peak correction method of heart sounds; however, it does not provide a noise reduction method for the inherent problems of heart sounds. Major heart sound problems, such as lung sounds, murmurs, respiration effects, and signal distortion in female subjects, were not addressed significantly in this paper.

\subsection{Concluding remarks}

In this study, only heart sound data were used to localize $S_{1}$ and $S_{2}$ features, with no other reference signal. This is a novel study in heart sound localization because it may provide an efficient and accurate method for heart sound applications. From the research presented, it appears that the proposed first and second heart sound localization method can improve localization performance. It may be a useful method for robust peak detection in various applications, such as LVET/PEP detection and cardiac disorder diagnoses. Although there are still unsolved problem related to abnormal heart activity and optimization for robust detection, we believe that the proposed method can provide simple and reliable heart sound feature

\section{Acknowledgements}

This work was supported by the Soonchunhyang University Research Fund (No. 20120676)

This research was supported by the MSIP(Ministry of Science, ICT and Future Planning), Korea, under the CITRC(Convergence Information Technology Research Center) (IITP-2015-IITP-2015-H8601-15-1009) supervised by the IITP(Institute for Information \& communications Technology Promotion)

\section{References}

[1] RP Lewis, SE Rittogers, WF Froester, and H Boudoulas, A critical review of the systolic time intervals, Circulation 56 (1977) 146-58.

[2] S Babaei and A Geranmayeh, Heart sound reproduction based on neural network classification of cardiac valve disorders using wavelet transforms of PCG signals, Comput Biol Med 39 (2009) 8-15.

[3] A Bartels and D Harder, Non-invasive determination of systolic blood pressure by heart sound pattern analysis, Clin Phys Physiol Meas 13 (1992) 249-56.

[4] XY Zhang, E MacPherson, and YT Zhang, Relations between the timing of the second heart sound and aortic blood pressure, IEEE Trans Biomed Eng 55 (2008) 1291-7.

[5] SM Debbal and F Bereksi-Reguig, Automatic measure of the split in the second cardiac sound by using the wavelet transform technique, Comput Biol Med 37 (2007) 269-76.

[6] SM Debbal and F Bereksi-Reguig, Computerized heart sounds analysis, Comput Biol Med 38 (2008) 263-80.

[7] M El-Segaier, O Lilja, S Lukkarinen, L Sornmo, R Sepponen, and E Pesonen, Computer-based detection and analysis of heart sound and murmur, Ann Biomed Eng 33 (2005) 937-42.

[8] C Gupta, R Palaniappan, S Swaminathan, and S Krishnan, Neural network classification of homomorphic segmented heart sounds, Applied Soft Computing Journal 7 (2007) 286-297.

[9] P Hult, T Fjallbrant, K Hilden, U Dahlstrom, B Wranne, and P Ask, Detection of the third heart sound using a tailored wavelet approach: method verification, Med Biol Eng Comput 43 (2005) 212-7.

[10] J Torry, Heart sound analysis comparing wavelet and autoregressive techniques, Computers in Cardiology (2003) 657-660.

[11] I Turkoglu, A Arslan, and E Ilkay, An intelligent system for diagnosis of the heart valve diseases with wavelet packet neural networks, Comput Biol Med 
33 (2003) 319-31.

[12] A Voss, A Mix, and T Hubner, Diagnosing aortic valve stenosis by parameter extraction of heart sound signals, Ann Biomed Eng 33 (2005) 1167-74.

[13] C Ahlstrom, A Johansson, F Uhlin, T Lanne, and P Ask, Noninvasive investigation of blood pressure changes using the pulse wave transit time: a novel approach in the monitoring of hemodialysis patients, J Artif Organs 8 (2005) 192-7.

[14] A Johansson, C Ahlstrom, T Lanne, and P Ask, Pulse wave transit time for monitoring respiration rate, Med Biol Eng Comput 44 (2006) 471-8.

[15] SJ Shah and AD Michaels, Hemodynamic correlates of the third heart sound and systolic time intervals, Congest Heart Fail 12 Suppl 1 (2006) 8-13.

[16] A Iwata, N Ishii, N Suzumura, and K Ikegaya, Algorithm for detecting the first and the second heart sounds by spectral tracking, Med Biol Eng Comput 18 (1980) 19-26.

[17] H Liang, S Lukkarinen, and I Hartimo, Heart sound segmentation algorithm based on heart sound envelogram, Computers in Cardiology (1997) 105108.

[18] A. Moukadem, A. Dieterlen, N. Hueber, C. Brandt, Comparative study of heart sounds localization, Proc. SPIE 8068, Bioelectronics, Biomedical, and Bioinspired Systems V; and Nanotechnology V, 80680P (2011) 99

[19] H Liang, S Lukkarinen, and I Hartimo, A boundary modification method for heart sound segmentation algorithm, Computers in Cardiology (1998) 593-595.

[20] T Oskiper and R Watrous, Detection of the first heart sound using a time-delay neural network, Computers in Cardiology (2002) 537-540.

[21] A Yadollahi and Z Moussavi, A robust method for heart sounds localization using lung sounds entropy, IEEE Transactions on Biomedical Engineering 53 (2006) 497-502.

[22] V Nigam and R Priemer, Accessing heart dynamics to estimate durations of heart sounds, Physiol Meas 26 (2005) 1005-1018.

[23] A Ricke, R Povinelli, and M Johnson, Automatic Segmenatation of Heart Sound Signals Using Hidden Markov Models, Computers in Cardiology 32 (2005) 953.

[24] S Messer, J Agzarian, and D Abbott, Optimal wavelet denoising for phonocardiograms, Microelectronics Journal 32 (2001) 931-941.

[25] P Wang, Y Kim, L Ling, and C Soh, First heart sound detection for phonocardiogram segmentation, Conf Proc IEEE Eng Med Biol Soc 5 (2005) 5519-22.

[26] C Ahlstrom, T Lanne, P Ask, and A Johansson, A method for accurate localization of the first heart sound and possible applications, Physiol Meas 29 (2008) 417-28.

[27] C Barabasa, M Jafari, A Robust Method for $S_{1} / S_{2}$
Heart Sounds Detection Without ECG Reference Based on Music Beat Tracking, Electronics and Telecommunications (ISETC), 2012 10th International Symposium (2012) 307-310.

[28] G Amit, K Shukha, N Gavriely, and N Intrator, Respiratory modulation of heart sound morphology, Am J Physiol Heart Circ Physiol 296 (2009) H796805 .

[29] A Haghighi-Mood and J Torry, A sub-band energy tracking algorithm for heart sound segmentation, Computers in Cardiology (1995) 501-504.

[30] T Kohama, S Nakamura, and H Hoshino, An efficient RR interval detection for ECG monitoring system, IEICE Transactions on Information and Systems E Series D 82 (1999) 1425-1432.

[31] H Baranek, H Lee, G Cloutier, and L Durand, Automatic detection of sounds and murmurs in patients with lonescu-Shiley aortic bioprostheses, Medical and Biological Engineering and Computing 27 (1989) 449-455.

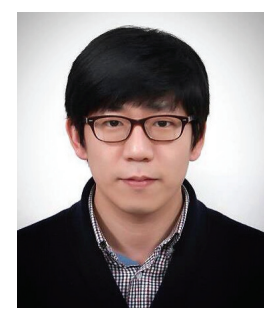

Se Dong Min He received the M.S. and Ph.D. degrees in electrical and electronic engineering from the Department of Electrical and Electronics Engineering, Yonsei University, Seoul, in 2004 and 2010, respectively. He is currently an Assistant Professor at the Department of Medical IT Engineering, Soonchunhyang University, Asan, Korea. His research area includes biomedical signal processing, healthcare sensor application, and mobile healthcare technologies.

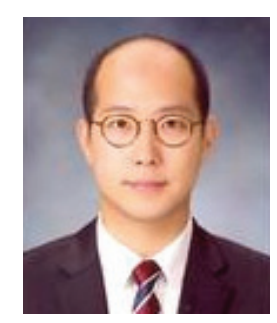

Hangsik Shin is the Assistant Professor at the Chonnam National University. $\mathrm{He}$ received the $\mathrm{Ph} . \mathrm{D}$. degree in electrical and electronic engineering from the departent of Electrical and Electronics Engineering, Yonsei University, in 2010. His research area include biomedical signal processing, physiological modeling, u-Healthcare and mobile Healthcare Technologies. 